son escogidos física e intelectualmente y además van y quieren ser capaces de superar sus metas, es comprensible. Pero no para el hombre o mujer de la calle que no posee estas condiciones, y que posiblemente ni tan siquiera disponga de todo el tiempo necesario para su adiestramiento. Además, el libro se refiere de una defensa contra armas, donde además del físico hay que tener la suficiente mente y sangre fría como para lanzarse a una defensa en la que si no se está preparado uno puede resultar muy lastimado o gravemente herido.

Pasemos a la faceta más positiva de la obra, un libro que procura ofrecer todos los consejos posibles para la seguridad, enfocándolo desde distintos ángulos. El primero como premisa docente, "No te lesiones", "Sé modesto", "Actúa correctamente", y "Conviértete en un experto, para no tener que matar".

En las posibles confrontaciones se aconseja, con buen criterio, que pueden ser evitadas, desapareciendo rápidamente de la escena. Como señala el dicho de un estratega japonés, "la batalla ganada es la no librada”, lógicamente cuando esto es posible, si no, como también aconseja el libro, cuando no hay más remedio "Si alguien viene a matarte, mátale tú primero". Como el libro va dirigido a profesionales y amantes de las artes marciales no creo, que deba "asustar" a otras personas.

El lector encontrará los fundamentos de defensa contra armas: una buena enumeración de sus principios básicos y las claves para su entreno, con fotos de calidad. La orientación, las estrategias y sus defensas están bien expuestas. Las defensas en la fase en que el agresor apuñala o acuchilla son claras y sencillas, empleando -menos en una acción- sólo golpes de puños, codos, rodilla, o pies en su defensa. Esto quiere decir que defenderse de armas peligrosas no debe ser algo complicado, debido al peligro que éstas conllevan.

El siguiente capítulo, cuando sólo es en principio una amenaza con el cuchillo pero que puede ser convertido en un ataque más peligroso, sus respuestas son como en el capitulo anterior. Por mi parte, echo de menos técnicas para saber llegar a un buen control del cuerpo del agresor y su arma. En el tercer capítulo el agresor está armado con un palo, ídem de lo anterior; al menos en la defensa contra armas el espíritu del creador era acabar con técnicas de golpeo.
Pasamos al siguiente capítulo en defensa contra pistola, aquí debido a la peligrosidad de la misma sí que llega al contacto y control para desarmar al agresor. Todas las técnicas están expuestas desde diferentes ángulos de ataque, desde cuchillo, palo y pistola.

El quinto capítulo es para especialistas en grupos de élite, pero puede interesar al lector civil, más inexperto porque en principio no tendrá conocimientos de los contenidos de defensa contra un rifle, escopeta o ametralladora. Aquí podrá informarse del contenido de cada arma (como suele decirse, para defenderte de algo primero tienes que conocerlo).

Siguiendo el mismo esquema anterior, con un alto grado de peligrosidad, cuando hay peligro de amenaza con una granada, en situaciones en que se recurre a la toma de rehenes. Interesante la forma de controlar, sujetar y desarmar dicha granada de su mano, sin que deba saltar la palanca y con ello explosionar el artefacto, incluye una breve explicación de su funcionamiento

En el capitulo siete se enseña a utilizar objetos cotidianos como armas defensivas, objetos que se pueden encontrar en cualquier momento inesperadamente y que pueden servir de defensa. Sigue con palo corto contra cuchillo, y después, palo contra palo. Hace referencias a los distintos métodos con palos desde el "método japonés", al "chino" e "inglés".

No se olvida en el capitulo diez la defensa contra dos agresores, con muy buenas recomendaciones y consejos. Tampoco de conceptos de evaluación, reacción y entrenamiento, y métodos de entrenamiento adicionales físico y mental. Personalmente, como profesional es lo que más aprecio y así se lo hago saber a los interesados en su introducción a las artes marciales: "Si no hay una mente dispuesta a evaluar lo que te están diciendo o enseñando, tu meta es corta”. Y aquí tienen un libro para profundizar en todos estos temas.

El libro acaba con la historia de los autores. Se trata, pues, de una obra real e interesante para cualquier amante de la autodefensa. Por lo que respecta a mi opinión sobre los controles, espero leer el libro de los autores en que la autodefensa se basa en el cuerpo a cuerpo.

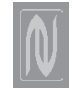

\section{Bruce Lee. Jeet Kune Do. Comentarios sobre el camino marcial}

Compilado por John Little

Badalona: Paidotribo, 2006

456 páginas. $21,5 \times 15 \mathrm{~cm}$. Ilustraciones

I.S.B.N.: 84-8019-860-5 • $34 €$

Disponible en:

Editorial Paidotribo

Polígono Les Guixeres

C/ de la Energía, 19-21

08915 Badalona (Barcelona - España)

Telf.: +34 933233311

Fax: + 34934535033

E-mail: paidotribo@paidotribo.com

http://www.paidotribo.com/

Revisión por Juan Pan González

En 1970, Bruce Lee sufrió una grave lesión de espalda que le mantuvo atado a una cama durante seis meses. Durante ese periodo, y fiel a la máxima de "convertir un obstáculo en un escalón”, comenzó a escribir acerca de sus creencias, filosofía, entrenamiento e ilustraciones sobre las artes marciales. Fruto de ese trabajo nació El Tao del Jeet Kune Do (Editorial Eyras, 1990). Sin embargo, muchas de sus notas y escritos no llegaron a ver la luz. En esta obra, Bruce Lee. Jeet Kune Do. Comentarios sobre el camino marcial, el autor John Little recupera gran cantidad de información acerca de la filosofía, técnicas y estrategias del combate, así como sobre la planificación de las lecciones y diversos comentarios de Bruce Lee acerca de temas diversos, que nunca llegaron a la luz pública.

La primera parte del libro consta de una serie de comentarios acerca de lo que Bruce Lee llamaba el "camino marcial", donde realiza una serie de reflexiones sobre el combate y los diversos estilos de artes marciales que estudió. Nos da una serie de breves ideas de lo que debería ser una artista marcial, su actitud, y del artista marcial como persona, y hace un pequeño análisis comparativo de los diferentes estilos de artes marciales, destacando los pros y contras en cuanto a la eficiencia en combate de estilos como el boxeo occidental, boxeo tailandés, karate, taekwondo, etc.

La segunda parte trata de los fundamentos del Jeet Kune Do, su origen, desarrollo, y los diferentes principios y estrategias que emplea. Da mucha 
importancia al dominio de la pierna y la mano adelantada, ya que el $80 \%$ de los golpes lanzados en un combate se realizan con la mano adelantada. Nos da las claves fundamentales a dominar para entrar en combate de una manera segura y eficiente, como son el control de la postura y el juego de piernas. Es un capitulo amplio y de interés para cualquier artista marcial, aunque en ocasiones la terminología empleada no está muy clara y emplea abreviaturas que aparecen conforme avanzamos en la lectura del libro, pero que en un primer momento nos dejan perdidos y sin saber su significado. Aparecen conceptos como ritmo roto, ligamentos y desligamientos, acometida, preparada, balance, desviaciones, etc., que no quedan muy claros y que dificultan la comprensión del texto si no se está familiarizado con ellos. La adecuada postura y el juego de piernas quedan explicados con detenimiento y de forma muy clara, llamando la atención en muchos aspectos que normalmente se descuidan y que son clave para tener éxito en un enfrentamiento.

La tercera y cuarta parte analizan los golpes más utilizados con las extremidades superiores e inferiores, y que para Bruce Lee eran más efectivos, sin embargo se hace un poco pesada su lectura dada su insistencia en determinados aspectos, así como en el planteamiento de todas y cada una de las posibilidades de cada golpe, a cada zona y desde las diferentes guardias de combate.

La quinta parte se centra en el entrenamiento de Jeet Kune Do, donde cabe destacar la visión que Bruce Lee tenia de las clases, los alumnos y el instructor, dando pautas muy útiles para cualquier profesional de las artes marciales. Aborda el tema de la estructura de las clases, la motivación de los alumnos, la influencia en el entrenamiento de la personalidad de cada alumno y la actitud del instructor en las clases, insistiendo en su profesionalidad y preparación. Posteriormente encontramos un plan básico de doce lecciones de Jeet Kune Do, un plan de diez lecciones que Bruce Lee llevaba a cabo con sus alumnos, y el plan de entrenamiento al que el propio Bruce se sometía a diario.

La última parte del libro expone gran cantidad de notas e ideas acerca del Jeet Kune Do como estilo de vida, sin duda él capitulo más profundo acerca de la vida, la existencia, la libertad del artista marcial, el arte marcial como camino de autoexpresión, el éxito, la actitud ante el fracaso, la conciencia, la fuerza de voluntad, el miedo... en definitiva, la manera en la que el arte marcial se relaciona con el ser humano.

Un libro que nos aproxima de forma profunda al Jeet Kune Do, su espíritu, filosofía y objetivos, donde el lector ha de implicarse con el material expuesto, estudiarlo y analizarlo, pero que, sobre todo, nos ayuda a comprender a Bruce Lee como artista marcial, como artista de la vida, siempre en proceso de creación continuo, para mejorar su proceso de crecimiento y autoexpresión a través de las artes marciales.

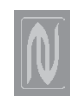

\section{T'ai Chi Ch'uan: Aspectos marciales. El arte del boxeo de sombras}

Por Kuo Lien-Ying

Versión de Guttman

Barcelona: La Liebre de Marzo, 2000

198 páginas. $21 \times 14 \mathrm{~cm}$. Ilustraciones

I.S.B.N.: 84-87403-44-1 • 13,52 €.

Disponible en:

La Liebre de Marzo

Arizala 1, entresuelo 1

08028 Barcelona (España)

Telf.: +34934485605

Fax: +34934498070

E-mail: espejo@liebremarzo.com

http://www.liebremarzo.com/

\section{Revisión por $\mathrm{M}^{\underline{a}}$ Teresa Gómez Alonso}

Este libro se acerca a un aspecto del T’ai Chi aún poco difundido en nuestro país como es su entrenamiento como arte marcial. La formación del alumno, en cuanto a la técnica física y la aplicación de estas habilidades marciales, no solamente exige conocer el lenguaje de los movimientos arquetípicos sino dominar un aspecto esencial del mismo que es la energía consciente. Explica los pasos necesarios para alcanzar la meta que es la maestría, y además muestra cómo valorar los progresos.

La recopilación, traducción y comentario de una colección de textos clásicos escritos en chino antiguo nos llevan a valorar esta obra de una forma especial. Kuo Lien-Ying (1895-1984), profesor de Guttmann y autor del libro, legó estos textos a sus alumnos, con el fin de preservar un sistema perfecto de arte marcial transmitido por generaciones anteriores. Kuo Lien-Ying en 1962 publicó un libro sobre el T'ai Chi, titulado Taijiquan pu (Crónica del T’ai Chi Ch'uan) que ha servido de base para la presente traducción.

El aspecto marcial del T’ai Chi va más allá de la práctica de movimientos circulares que coordinan brazos, piernas y mirada a modo de danza. La aplicación real es dura, rápida, y el objetivo es derrotar al contrario a la vez que se trabaja el crecimiento personal.

En la introducción y el primer capitulo el autor nos muestra los conceptos básicos del T'ai Chi como arte marcial (el arte del boxeo de sombras). Comienza con un estudio de la intersecciónoposición entre el Yin y el Yang: "Al comprender el concepto del yin y yang, podemos expresar el Tao, podemos comprender el cielo, la tierra... son dos, hay que combinarlos para que se conviertan en uno. Uno puede ser dos, y dos pueden ser uno, a los que se conoce como los tres: Cielo, Tierra y Humanidad" (p.18). Para continuar con los concepto esenciales de la lucha como son: la energía consciente, el equilibrio, la calma, la acción y los principios de la lucha (lo débil supera a lo fuerte y lo lento a lo rápido). Todo ello con un lenguaje poético que pretende ilustrar la complejidad de los conceptos, evocando metafóricamente la naturaleza, y el cual se va a repetir a lo largo del libro.

El capitulo dos nos introduce en la complejidad del T'ai Chi como arte marcial, deteniéndose en los detalles, en la acción y en la disposición de cuerpo en general y de sus diferentes partes en particular. Desde una perspectiva pedagógica secuenciada desarrolla tres pasos. El primero investiga "el cuerpo, la cintura, la parte superior de la cabeza, la columna, los pasos, y la forma ("La crónica del arte marcial dice que la forma del practicante debe ser como un halcón que atrapa un conejo, y el espíritu como el de un gato cazando un ratón")" (p.36). El segundo estudia las cuatro categorías de movimiento de energía: movilizar, recibir, coger y atacar. Y el tercero pone en práctica los pasos anteriores en la acción motriz de la lucha, así estudia: el seguir como una línea de conexión entre las extremidades, la ligereza y la agilidad. A partir de este capitulo el autor introduce en cada apartado preguntas 